\title{
$0.395 \mathrm{THz}$ Surface Wave Oscillator for DNP-NMR Applications
}

\author{
Amy MacLachlan, Craig Robertson, Adrian Cross and Alan Phelps \\ Department of Physics, SUPA, University of Strathclyde \\ Glasgow, Scotland, UK, G4 0NG \\ amy.maclachlan@strath.ac.uk
}

\begin{abstract}
The simulation and design of an oversized, twodimensional, periodic surface lattice (2D PSL) interaction cavity used within a $0.395 \mathrm{THz}$, pulsed radiation source is presented. Powerful, pulsed radiation with good spectral purity is demonstrated. The device is well-suited for use in DNP-NMR spectroscopy and has numerous other applications due to the scalability of the interaction cavity allowing for radiation output at different frequencies. The device exploits the coupling of volume and surface fields in the 2D PSL interaction cavity to excite a single cavity eigenmode. The observed output power is $13.5 \mathrm{~kW}$ and the efficiency of the device is $27 \%$
\end{abstract}

Keywords - terahertz, surface field, mode coupling, highpower, oscillator, DNP-NMR

\section{INTRODUCTION}

Powerful, coherent terahertz sources have applications in wireless communications, non-destructive testing, environmental sensing, $\mathrm{THz}$ spectroscopy, medicine and plasma diagnostics. Radiation sources at certain frequencies, including $395 \mathrm{GHz}$, are required for enhancing NMR using the DNP-NMR method, for which powers of around 50$100 \mathrm{~W}$ are typically needed. The power required for DNPNMR exceeds the maximum power that can be delivered by solid state sources at $\mathrm{mm}$-wave and $\mathrm{THz}$ frequencies. The proposed $0.395 \mathrm{THz}$ surface wave oscillator, based on a twodimensional, cylindrical periodic surface lattice (2D PSL) interaction cavity can be exploited as a potentially more cost effective alternative to gyrotrons for DNP-NMR applications.

The theory and concepts presented in this paper are scalable and the 2D PSL surface wave oscillator can be engineered to operate at different frequencies including 263 $\mathrm{GHz}, 527 \mathrm{GHz}$ and $593 \mathrm{GHz}$ which are also relevant for DNP-NMR. It should be noted that, whilst the same theoretical and numerical methods [1-8] can be used to design the 2D PSLs at higher frequencies, manufacturing a 2D PSL designed to operate at $595 \mathrm{GHz}$ to within an acceptable tolerance is extremely challenging due to the shorter corrugation period associated with the wavelength.

Cylindrical 2D PSLs fabricated by creating a shallow, periodic corrugation on the inner surface of a cylindrical waveguide have been constructed using additive manufacturing [3,9], or by copper electrodeposition. High precision laser etching has also been used previously to make a planar 2D PSL with a period of $0.64 \mathrm{~mm} \mathrm{[6].} \mathrm{With}$ continuing advances in high-precision fabrication capabilities [10] and nanotechnology, it is hoped that very high frequency interaction cavities, even above $0.5 \mathrm{THz}$, will soon become feasible, allowing for the realization of 2D PSL surface wave oscillators further into the $\mathrm{THz}$ regime.

High power radiation is generated by guiding a thin, annular electron beam close to the corrugated inner wall of

This work has been supported by the AFOSR award numbers FA8655-131-2132 and FA9550-17-1-0095. the cylindrical 2D PSL [1-8]. In this paper, the localized surface field, bound to the inner corrugated wall of the 2D PSL, facilitates the production of radiation at $0.395 \mathrm{THz}$. The lattice corrugation is shallow in comparison to the radius, and the cavity has similar electromagnetic properties to a smooth cylindrical waveguide loaded with a thin dielectric layer. A novel radiation source that exploits surface polaritons to generate Cherenkov radiation is demonstrated in [11].

The device demonstrated in this work is a type of oversized surface wave oscillator, and the diameter D of the interaction cavity is scaled up in size so that the ratio $D / \lambda$ is several times the free space wavelength. Compared to other radiation sources, where the output power is restricted by the diameter, especially at shorter wavelengths, a source incorporating an oversized, cylindrical 2D PSL, in contrast to a 1D PSL [12], is capable of producing higher output power at sub $\mathrm{THz}$ and $\mathrm{THz}$ frequencies. The increased cavity size results in overmoding which would ordinarily lead to multimode excitation. However, it is possible to excite a single, coupled cavity eigenmode if the close to cut-off, azimuthally symmetric, transverse magnetic (TM) volume field and surface fields within the cavity are coupled. By employing this novel mode coupling technique, high power radiation with good spectral purity can be observed.

To excite the cavity eigenmode, composed of coupled volume and surface fields, the 2D PSL must be precisely engineered. Even subtle changes to the optimum parameters and dimensions can affect the operation of the device. We present the design of a $0.395 \mathrm{THz}$ radiation source as well as the results of numerical simulations carried out using the particle in cell (PIC) code solver of CST Microwave Studio (CST MWS).

The $0.395 \mathrm{THz}, 2 \mathrm{D}$ PSL used to demonstrate the fundamental, "proof-of-principle" mode selection in a large cavity, has a diameter to wavelength ratio of $D / \lambda=2.8$. Cavities with much larger $D / \lambda$ values are theoretically possible and a 2D PSL with $D / \lambda \sim 5$ and an operating frequency of approximately $0.1 \mathrm{THz}$ has been manufactured using electrodeposition and deployed within an ongoing experiment at the University of Strathclyde.

\section{THEORY AND DESIGN}

The amplitude of the 2D PSL corrugation is much smaller than the operating wavelength, and the 2D PSL can therefore be described as an "effective meta-dielectric". The 2D periodic corrugation along the axial and azimuthal directions is described by the equation

$$
r=r_{0}+\Delta r \cos \left(k_{z} z\right) \cos (\bar{m} \varphi)
$$

where $r_{0}$ is the mean radius, $\Delta r$ is the amplitude of the corrugation, and $\bar{m}$ is the number of azimuthal variations. 
For coupling between the electron beam and the coupled eigenmode of the 2D PSL for low-voltage (mildly relativistic) electron beams the following criterion must be satisfied.

$$
\bar{m} \leq 2 \pi r_{0} / \lambda \gamma
$$

where $\gamma$ is the relativistic Lorentz factor. The azimuthal period is $d_{m}=2 \pi r_{0} / \bar{m}$ and the axial period $d_{z}=2 \pi / k_{z}$ is shortened to facilitate coupling with the electron beam.

A tapered period length at both the input and output ends of the structure is incorporated into the design of the interaction structure. The total length is $L=(n+2) d_{z}$ where $n$ is the number of longitudinal periods of the 2D PSL. The radius $r_{0}$ of the cylinder is chosen to be close to the cutoff frequency of the azimuthally symmetric, $\mathrm{TM}_{0,3}$ volume mode involved in the formation of the coupled cavity eigenmode. The parameters of the $0.395 \mathrm{THz} 2 \mathrm{D}$ PSL are provided in Table 1 and a diagram showing the 2D PSL, taper and cathode configuration is presented in Fig.1.

A planar grid structure (simulated as a transparent, perfect electrical conductor (PEC) grid in CST MWS) is positioned between the flat cathode and the input end of the 2D PSL structure, as shown in Fig.1. The planar grid greatly enhances the quality of the electron beam and reduces the amount of current, and therefore input power, needed to drive the device. The parameters of the 2D PSL have been chosen to be compatible with a $50 \mathrm{kV}$ electron beam, and the use of the grid allows for a relatively low beam current of $1 \mathrm{~A}$. The thin, annular electron beam must be close to the corrugated wall in order to intercept the exponentially decaying surface field.

TABLE I. PARAMETERS OF 2D PSL

\begin{tabular}{|l|c|c|c|c|}
\hline \multicolumn{5}{|c|}{ Parameters of 0.396 THz 2D PSL } \\
\hline $\boldsymbol{r}_{\mathbf{0}}(\mathbf{m m})$ & $\overline{\mathbf{m}}$ & $\boldsymbol{d}_{\mathbf{z}}(\mathbf{m m})$ & $\boldsymbol{\Delta} \boldsymbol{r}(\mathbf{m m})$ & $\boldsymbol{n}$ \\
\hline 1.058 & 7 & 0.32 & 0.06 & 8 \\
\hline
\end{tabular}

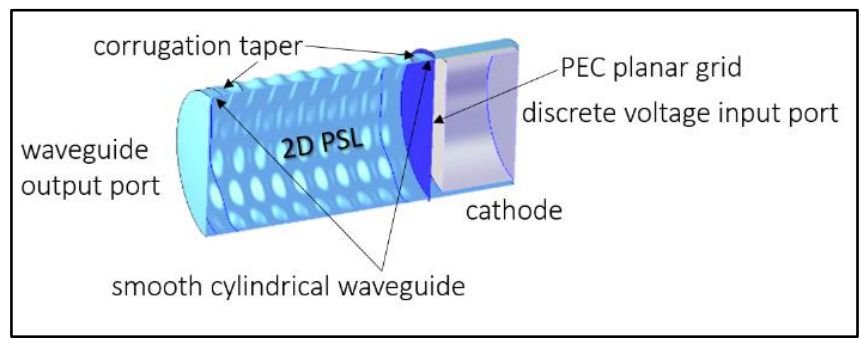

Fig. 1. Diagram showing the 2D PSL, and cathode set-up as simulated using the PIC solver of CST MWS

\section{Simulation Results}

The CST MWS simulations were run over an electron beam duration of 30ns and calculations were made for 63 modes. The high quality of the electron beam is maintained after $27 \mathrm{~ns}$ as shown in Fig.2. The electron beam is immersed in a strong magnetic field of $5 \mathrm{~T}$ which maintains the separation of $0.1 \mathrm{~mm}$ between the outer edge of the beam and the outer radius of the 2D PSL corrugation. Fig. 3 shows the radiation output, plotted on a $\mathrm{dB}$ scale, and over a frequency range of $300-500 \mathrm{GHz}$. A powerful signal is observed at $0.395 \mathrm{THz}$ and the spectral purity demonstrates the effectiveness of the novel mode coupling technique used in this work.

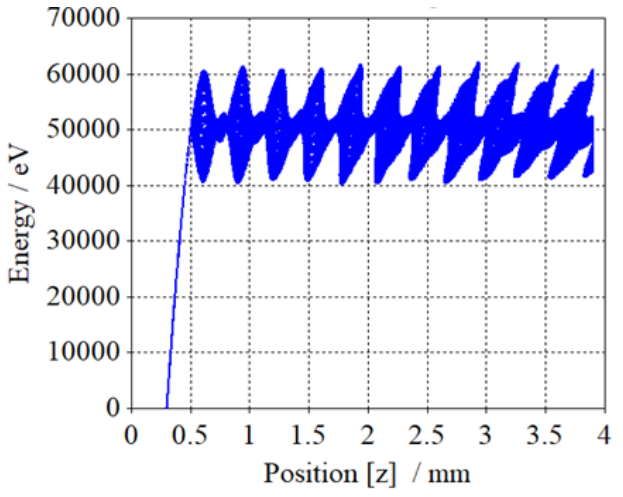

Fig. 2. CST MWS simulation results showing the high quality, $50 \mathrm{kV}$ electron beam achieved by using the planar grid with a strong, $5 \mathrm{~T}$ magnetic field.

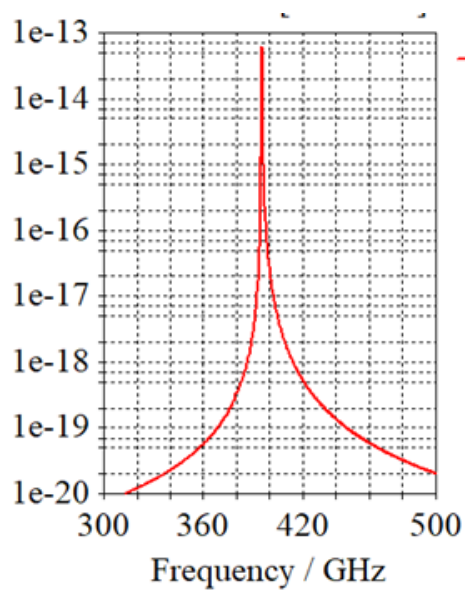

Fig. 3. CST MWS results showing the cavity eigenmode signal at 0.395 $\mathrm{THz}$ and high spectral purity of the device.

Contour plots of the axial field components of the $\mathrm{TM}_{0,3}$ volume mode and the coupled cavity eigenmode at $0.395 \mathrm{THz}$ are presented in Fig.4. The axial electric field component of the cavity eigenmode (Fig.4 (a)) resembles a $\mathrm{TM}_{5,1}$ mode. The presence of a small, axial magnetic field component with an azimuthal index of 7 (Fig.4(b)) suggests that the eigenmode is slightly hybrid. This small $\mathrm{H}_{\mathrm{z}}$ field component is similar to a $\mathrm{EH}_{7,1}$ surface field and the azimuthal index of the surface field $m_{s}$ corresponds to the number of azimuthal variations of the structure, $\bar{m}=m_{s}$ as required to satisfy the azimuthal Bragg resonance condition $[1,2,5,7]$.
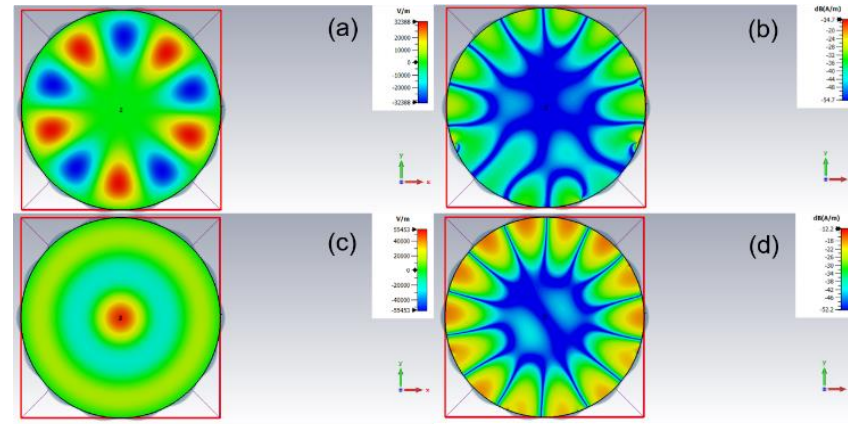

Fig. 4. Contour plots showing the axial electric (left) and magnetic (right) field components of the coupled cavity eigenmode $(a, b)$ and the near-cut off $\mathrm{TM}_{0,3}$ volume mode (c,d). Both the coupled cavity eigenmode and the $\mathrm{TM}_{0,3}$ volume mode have a small axial magnetic field component with an azimuthal index of 7 . 
The cavity eigenmode is the $40^{\text {th }}$ mode in the CST MWS mode index, just one mode above the $\mathrm{TM}_{0,3}$ volume mode, which is mode 39 in the CST MWS results. Fig. 4(d) shows that the $\mathrm{TM}_{0,3}$ volume mode also has a small $\mathrm{H}_{\mathrm{z}}$ field component with an azimuthal index of 7 , suggesting that the $\mathrm{TM}_{0,3}$ volume mode is resonantly coupled with the cavity eigenmode.

The CST MWS results show $13.5 \mathrm{~kW}$ of output power in the coupled cavity eigenmode, giving an output efficiency of $27 \%$. There is no significant power in any of the other modes.

\section{CONCLUSIONS}

The work presented in this paper demonstrates the production of $0.395 \mathrm{THz}$, pulsed radiation with an output power and frequency well suited to DNP NMR applications. The device is scalable to different frequencies and the output power capabilities can be increased for other applications by making the 2D PSL interaction cavity more oversized, by exploiting the method of volume and surface field coupling to achieve $D / \lambda \geq 10$.

\section{REFERENCES}

[1] I. V. Konoplev, A. J. MacLachlan, C. W. Robertson, A. W. Cross and A. D. R. Phelps, "Cylindrical periodic surface lattice as a metadielectric: concept of a surface-field Cherenkov source of coherent radiation”, Phys. Rev. A, vol. 84, 013836, July 2011.

[2] I. V. Konoplev, A. J. MacLachlan, C. W. Robertson, A. W. Cross and A. D. R. Phelps, "Cylindrical, periodic surface lattice - theory, dispersion analysis and experiment", Appl. Phys. Lett., vol. 101, 121111, Sept. 2012.

[3] A. R. Phipps, A. J. MacLachlan, C. W. Robertson, L. Zhang, I. V. Konoplev, A. W. Cross and A. D. R. Phelps, "Electron beam excitation of coherent sub-terahertz radiation in periodic structures manufactured by 3D printing", Nucl. Instrum. Methods Phys. Res. Sect. B, vol. 402, 202-205, July 2017.

[4] A. J. MacLachlan, C.W. Robertson, A.W. Cross and A. D. R. Phelps, "Volume and surface mode coupling experiments in periodic surface structures for use in mm-THz high power radiation sources", AIP Advances, vol. 8, 105115, Oct. 2018.

[5] A. J. MacLachlan, C. W. Robertson, I. V. Konoplev, A. W. Cross, A. D. R. Phelps and K. Ronald, "Resonant excitation of volume and surface fields on complex electrodynamic surfaces", Phys. Rev. Appl., vol. 11, 034034, March 2019.

[6] A. J. MacLachlan, C. W. Robertson, K. Ronald, A. W. Cross and A. D. R. Phelps, "Mode coupling in periodic surface lattice and metamaterial structures for mm-wave and THz applications", SN Appl. Sciences, vol. 1, 613, June 2019.

[7] A. J. MacLachlan, C. W. Robertson, A. W. Cross, K. Ronald and A. D. R. Phelps, "Excitation and coupling of volume and surface fields on complex electrodynamic surfaces at mm-wave and THz frequencies", IET Microw. Antennas Propag., vol. 14, (11), pp. 1151-1156, May 2020.

[8] N. S. Ginzburg, E. V. Ilyakov, I. S. Kulagin, A. M. Malkin, N. Y. Peskov, A. S. Sergeev and V. Y. Zaslavsky, "Theoretical and experimental studies of relativistic oversized Ka-band surface-wave oscillator based on 2D periodical corrugated structure", Phys. Rev. Accel. and Beams, vol. 21, 080701, Aug. 2018.

[9] D. M. French and D. Shiffler, "High power microwave source with a three dimensional printed metamaterial slow-wave structure", Rev. Sci. Instrum., vol. 87, 053308, May 2016.

[10] J. Feng, Y. Tang, D. Gamzina, X. Li, B. Popovic, M. Gonzalez, L. Himes, R. Barchfeld, H. Li, P. Pan, R. Letizia, C. Paoloni, and N. C. Luhmann, "Fabrication of a 0.346-THz BWO for Plasma Diagnostics", IEEE Trans. Electron Devices, vol. 65, pp. 2156-2163, April 2018.

[11] S. Liu, P. Zhang, W. Liu, S. Gong, R. Zhong, Y. Zhang, and M. Hu, "Surface Polariton Cherenkov Light Radiation Source", Phys. Rev. Lett., vol. 109, 153902, Oct. 2012.

[12] I. V. Konoplev, P. McGrane, A. D. R. Phelps, A. W. Cross, K. Ronald, "Observation of photonic band-gap control in one-dimensional Bragg structures", Appl. Phys. Lett., vol. 87, 121104, Sept. 2005. 